\section{(C) OPEN ACCESS}

\title{
Timely access to care for patients with critical burns in India: a prehospital prospective observational study
}

\author{
Jennifer A Newberry, ${ }^{1}$ Corey B Bills, ${ }^{2}$ Elizabeth A Pirrotta, ${ }^{1}$ Michele Barry, ${ }^{3}$ \\ Govindaraju Venkata Ramana Rao, ${ }^{4}$ Swaminatha V Mahadevan, ${ }^{1}$ \\ Matthew C Strehlow ${ }^{1}$
}

\begin{abstract}
'Department of Emergency Medicine, Stanford University School of Medicine, Stanford, California, USA

${ }^{2}$ Emergency Medicine, University of California San Francisco School of Medicine, San Francisco, California, USA Internal Medicine, Stanford University School of Medicine, Stanford, California, USA

${ }^{4}$ GVK Emergency Management and Research Institute,

Hyderabad, India
\end{abstract}

\section{Correspondence to} Dr. Jennifer A Newberry, Department of Emergency Medicine, Stanford University School of Medicine, Stanford, CA 94034, USA newberry@stanford.edu

Received 20 June 2018 Revised 7 December 2018 Accepted 20 December 2018 Published Online First 11 January 2019

\begin{abstract}
Background Low/middle-income countries carry a disproportionate burden of the morbidity and mortality from thermal burns. Nearly $70 \%$ of burn deaths worldwide are from thermal burns in India. Delays to medical care are commonplace and an important predictor of outcomes. We sought to understand the role of emergency medical services (EMS) as part of the healthcare infrastructure for thermal burns in India.

Methods We conducted a prospective observational study of patients using EMS for thermal burns across five Indian states from May to August 2015. Our primary outcome was mortality at 2, 7 and 30 days. We compared observed mortality with expected mortality using the revised Baux score. We used $\mathrm{X}^{2}$ analysis for categorical variables and Wilcoxon two-sample test for continuous variables. ORs and $95 \% \mathrm{Cls}$ are reported for all modelled predictor variables.
\end{abstract}

Results We enrolled 439 patients. The 30-day follow-up rate was $85.9 \%(n=377)$. The median age was 30 years; $56.7 \%(n=249)$ lived in poverty; and $65.6 \%(n=288)$ were women. EMS transported $94.3 \%$ of patients $(n=399)$ to the hospital within 2 hours of their call. Median total body surface area (TBSA) burned was $60 \%$ overall, and $80 \%$ in non-accidental burns. Sixty-eight per cent of patients had revised Baux scores greater than 80. Overall 30 -day mortality was $64.5 \%$, and highest $(90.2 \%)$ in women with non-accidental burns. Predictors of mortality by multivariate regression were TBSA (OR 7.9), inhalation injury (OR 5.5), intentionality (OR 4.7) and gender (OR 2.2).

Discussion Although EMS rapidly connects critically burned patients to care in India, mortality remains high, with women disproportionally suffering self-inflicted burns. To combat the burn epidemic in India, efforts must focus on rapid medical care and critical care services, and on a burn prevention strategy that includes mental health and gender-based violence support services.

\section{BACKGROUND}

Globally, there were over 33.5 million thermal burn injuries in 2013, resulting in over 1 million years lost to disability, and approximately 237500 deaths. ${ }^{2}$ The majority of burn deaths, an estimated $90 \%$, occur in low/middle-income countries (LMIC). ${ }^{3}$ Burns remain a significant public health problem in LMICs, contributing to substantial morbidity and mortality.

A review of literature on burns in LMICs reported an overall mortality rate of $9.9 \%$, reaching

\section{What is already known on this subject}

- Low- and middle-income countries carry a disproportionate burden of the morbidity and mortality from fire-related burns.

- Improvements in Emergency Medical Services (EMS) can potentially result in faster treatment.

- The outcome for patients using EMS has not been studied.

\section{What this study adds}

- In this prospective observational study, patients in India who used EMS for critical burn injuries were transported quickly to hospitals, with $88 \%$ of patients reaching hospitals within three hours of the original injury.

- The most critically ill patients cared for by this EMS system were women with non-accidental burns - this cohort had a $90.2 \%$ mortality rate.

as high as $21.3 \%$ in hospitalised patients. ${ }^{4}$ In these studies, most burns covered less than $10 \%$ total body surface area (TBSA). ${ }^{4}$ However, in India, where an estimated 163000 deaths occur annually due to fire-related injuries, the patterns of burns are different..$^{5}$ In a study of 222 patients admitted to a tertiary care centre in India, the average TBSA of the 135 patients who died was $65.7 \%$ (SD 2.1\%). In this study, TBSA, gender, age and delays in accessing or receiving care were associated with a statistically significant increased risk of death. A large retrospective study of 5 years of autopsy data from Lucknow reported similar observations. ${ }^{7}$ Of their 2225 records reviewed, $87.5 \%$ were female. Of those with self-inflicted burns, almost $40 \%$ suffered burns of over $80 \%$ TBSA. Compared with other LMICs, India faces the highest number of self-inflicted burns, which are also associated with the highest fatality rate. ${ }^{8}$

Emergency medical services (EMS) may play a key role in the public health infrastructure to improve outcomes from burns. EMS could decrease time to care and increase access to care, particularly specialised critical care services. However, there is only a single retrospective study on burns seen by EMS in LMICs $^{9}$ and no research in India specifically examining the epidemiology of patients with burns seen by EMS. In this study, we sought to characterise the 
demographics and outcomes of patients using EMS for thermal burns in India.

\section{METHODS}

We conducted a prospective observational study of patients using a single EMS system for thermal injuries across five states in India-Andhra Pradesh, Assam, Gujarat, Karnataka and Telangana. EMS was established in these states between 2005 and 2008. A convenience sample of patients were enrolled Monday through Saturday during daytime hours, due to research assistant availability and safety concerns on night shifts. Enrolment occurred over 4 months from May to August 2015.

\section{Setting}

GVK Emergency Management Research Institute (GVK EMRI) operates EMS across 15 states and union territories in India, covering over 750 million people. In these states, individuals have access to free prehospital emergency medical care and ambulance transport via a toll-free phone number, 108. Ambulances are staffed by a driver and a single emergency medical technician (EMT). EMTs are trained to provide wound care and resuscitation, including basic airway management and intravenous fluids. Following initial assessment and treatment, ambulances transport patients to the nearest hospital, unless otherwise requested by the patient or family. As this EMS system is operated by a public-private partnership, most patients are taken to a government hospital. Government hospitals can be generally categorised by the level of care they are built to provide: primary (eg, primary health centres), secondary (eg, community health centres, district hospitals) and tertiary (eg, medical colleges). There is variability even within these divisions, but intensive care capabilities and specialty burn care would only be available at tertiary centres.

\section{Study design}

Any patient calling 108 for a burn injury was eligible for inclusion. Exclusion criteria included calls for burns caused primarily by chemicals or electricity, interfacility transfers, patients who were absent on EMT arrival and patients who refused care and transport. At initial enrolment, trained research assistants used a standardised questionnaire to collect data in real time by phone from EMTs. Data included demographics, medical history, physical exam, care rendered by the EMT and whether the injury was accidental or non-accidental (per patient or caller report). In order to limit the number of patients lost to follow-up, research assistants collected two phone numbers at the time of initial patient enrolment. All data were securely collected and managed via REDCap (Stanford University). ${ }^{10}$

Follow-up phone calls were completed at 2, 7 and 30 days. Follow-up data collection included patient status, hospital admission and functional status. Per GVK EMRI's standard procedures, participants provided verbal consent for medical care, transport and follow-up at the time of their enrolment.

Our primary outcome was mortality at 2, 7 and 30 days. We compared observed mortality with expected mortality using the revised Baux score (age $+\%$ TBSA +17 (if inhalation injury)). ${ }^{11}$ The revised Baux score has been shown to perform well in another LMIC and a score of 80 translated to a 50\% fatality rate $\left(\mathrm{LD}_{50}\right) .{ }^{12}$ Secondary outcomes included oxygen delivery for suspected inhalation injury, intravenous fluids for hypotension and functional status. Suspected inhalation injury was defined as the presence of facial burns, soot in the nares or mouth, hoarse voice, increased work of breathing, stridor or wheezing. Hypotension was defined as systolic BP $<90 \mathrm{~mm} \mathrm{Hg}$. In follow-up, we asked patients to rate their functional status, ranging from a complete return to normal activities to being bedridden by pain or injury.

For missing data, we used complete case analysis for comparative analysis. We used $\mathrm{X}^{2}$ analysis for categorical variables (or Fisher's exact test when appropriate) and Wilcoxon two-sample test for continuous variables. A p value $<0.05$ was considered statistically significant. We report ORs and 95\% CIs for all modelled predictor variables. For multivariate regression, we model the components of the Baux scores as individual predictors as this model has not been studied in India yet. Further, we model TBSA as a binary variable rather than continuous. Specifically, we chose a cut-off of 10\% TBSA, as these are typically transferred to a burn centre in the USA due to increased morbidity and mortality. All data analyses were conducted via SAS Enterprise Guide for Windows, V.7.15 (SAS Institute).

\section{RESULTS}

From our initial sample of 614 calls, we excluded 154 calls (25.1\%) for electrical burns and 21 calls $(3.4 \%)$ without a clear aetiology. Our final enrolment of 439 individuals represents $11.4 \%$ of all calls for burns across these states during the study. We may be underestimating our representativeness of calls for thermal burns specifically because burn type is not identified at dispatchFigure 1.

The median age was 30 (IQR 22-40), with only $13.4 \%(n=59)$ below the age of 18 , and $5.7 \%(n=25)$ aged 65 or older (table 1$)$. Patients were predominately female $(n=288 ; 65.6 \%)$ and from primarily rural and tribal areas $(n=318 ; 72.4 \%)$. Certain land in India is designated as protected for tribal peoples. These areas tend to have poorer infrastructure and lower population density than rural areas.

Ninety-one percent of calls were made within 1 hour of injury $(n=398)$, with most occurring within 30 min of injury $(n=346$; $78.8 \%)$. Once a call was placed, the median time from call to hospital arrival was $56 \mathrm{~min}$ (IQR 35-81 min). Ambulances transported most patients to hospitals within 2 hours of their call $(\mathrm{n}=399 ; 94.3 \%)$. Transport from rural areas, $66 \mathrm{~min}$ (IQR 45-88), was significantly longer than from urban areas, $35 \mathrm{~min}$ (IQR 26-57; p <0.01); however, the median distance travelled for rural calls, $17 \mathrm{~km}$ (IQR 10-30), was nearly three times farther than for urban calls, $6 \mathrm{~km}$ (IQR 3-12). Transport distances and times from tribal areas (20 km (IQR 10-32), $64 \mathrm{~min}$ (IQR 39-86)) were similar to rural areas. Patients who died prior to EMT arrival $(n=20)$ were not transported. Most patients who were transported were taken to government hospitals $(n=377$; $90.0 \%)$. Fifty-nine per cent $(n=247)$ were transported to a secondary care centre (eg, community hospital centre or district hospital), while $27.4 \%(n=115)$ were transported to a tertiary care centre (eg, medical college).

Of the 419 patients who were alive at the time of EMT arrival, $81.6 \%(n=342)$ had at least three of the following four key vital signs measured by the EMT: BP, pulse, RR and oxygen saturation (table 2). EMTs reported that for 80.9\% $(n=110)$ of patients without a measured BP, a measurement was not taken because patients had sustained burns to both arms. Other reasons included non-functional equipment $(8.8 \%, \mathrm{n}=12)$; the EMT believed it was not indicated $(4.4 \% ; n=6)$; and lack of time $(2.9 \% ; n=4)$. In $91.0 \%(n=111)$ of cases where EMTs did not measure an oxygen saturation, the reason was a lack of functional equipment. 


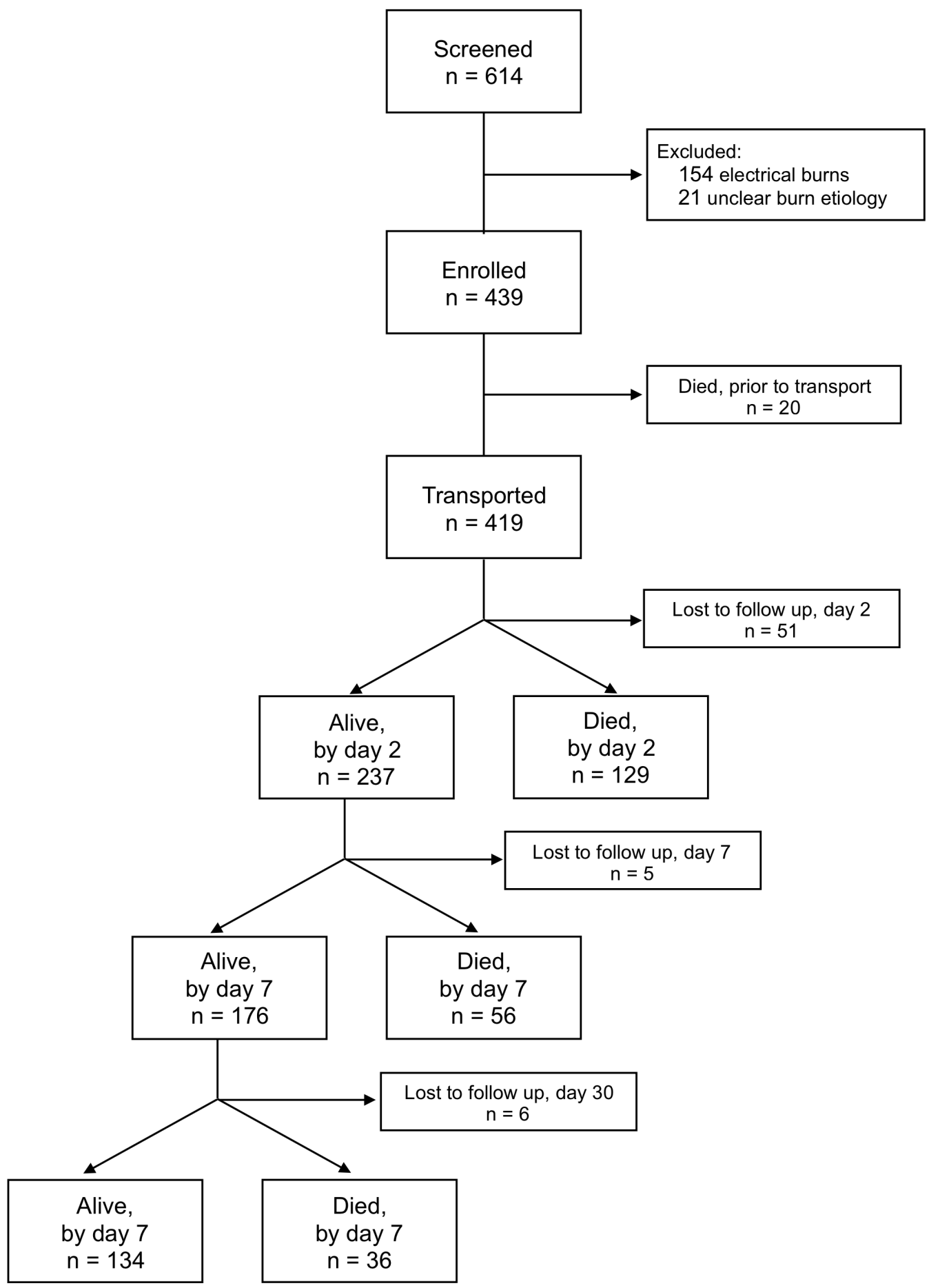

Figure 1 Flow diagram of patient study cohort.

Almost three-quarters of all patients had evidence of inhalation injury $(69.7 \% ; n=306)$. Of patients with suspected inhalation injury who were alive on EMT arrival, 83.0\% $(n=240)$ received oxygen. Further, $95.6 \%$ of patients with noted hypoxia (oxygen saturation <90\%) were given oxygen by EMTs. Only 25 patients $(5.7 \%)$ were hypotensive, and 12 of these patients were given intravenous fluids (48.0\%).

The median EMT estimation of total TBSA burned was $60 \%$ (IQR: 30\%-90\%) (table 3). Younger patients (less than 18 years old) had a significantly lower TBSA burned compared with all other age groups $(25 \%$; IQR: $13 \%-80 \%$; $<<0.01)$. Across ages, women had significantly higher TBSA burned than men, $70 \%$ vs $36 \%(\mathrm{p}<0.01)$. Two hundred and five patients (or their families) reported non-accidental burns (46.7\%), most of which were reported to be self-inflicted $(97.6 \% ; n=200)$. The median TBSA of non-accidental burns was significantly greater than accidental burns $(80 \%$ vs $35 \% ; \mathrm{p}<0.01)$. Comparing women and men, even for accidental burns women had a significantly higher median TBSA ( $40 \%$ vs $25 \%$; $<<0.01$ ).

Response rates at 2,7 and 30 days were $88.4 \%, 87.2 \%$ and $85.9 \%$, respectively. Most patients $(93.4 \% ; n=342)$ were admitted for at least one night to a hospital and 16.9\% $(n=62)$ reported transfer to a higher level of care at some point. Of patients alive at 30 days, only $11.2 \%(n=15)$ reported being 
Table 1 Demographics of patients using EMS for burn injuries across five states in India

\begin{tabular}{|c|c|c|}
\hline Characteristic* & $\mathbf{n}$ & $\%$ \\
\hline All & 439 & 100 \\
\hline \multicolumn{3}{|l|}{ State } \\
\hline Andhra Pradesh & 34 & 7.7 \\
\hline Assam & 23 & 5.2 \\
\hline Gujarat & 169 & 38.5 \\
\hline Karnataka & 136 & 31.0 \\
\hline Telangana & 77 & 17.5 \\
\hline \multicolumn{3}{|l|}{ Age (years) } \\
\hline$<18$ & 59 & 13.4 \\
\hline $18-24$ & 81 & 18.5 \\
\hline $25-34$ & 119 & 27.1 \\
\hline $35-44$ & 84 & 19.1 \\
\hline $45-54$ & 55 & 12.5 \\
\hline $54-64$ & 16 & 3.6 \\
\hline$\geq 65$ & 25 & 5.7 \\
\hline \multicolumn{3}{|l|}{ Gender } \\
\hline Female & 288 & 65.6 \\
\hline Male & 151 & 34.4 \\
\hline \multicolumn{3}{|l|}{ Marital status } \\
\hline Married & 327 & 74.5 \\
\hline Unmarried & 67 & 15.3 \\
\hline \multicolumn{3}{|l|}{ Economic status } \\
\hline Below poverty level & 249 & 56.7 \\
\hline Above poverty level & 182 & 41.5 \\
\hline \multicolumn{3}{|l|}{ Incident location } \\
\hline Urban & 120 & 27.3 \\
\hline Rural & 280 & 63.8 \\
\hline Tribal & 38 & 8.7 \\
\hline
\end{tabular}

*Missing data: marital status $(n=44)$, economic status $(n=16)$, incident location $(n=1)$.

EMS, emergency medical services; TBSA, total body surface area.

bedridden or unable to resume normal activities due to pain. An additional 35.8\% $(n=48)$ remained in pain, but felt able to complete their normal activities.

Table 2 EMT assessment and interventions performed for patients with burns across five states in India

\begin{tabular}{lcc}
\hline EMT performance & $\mathbf{n}$ & $\%$ \\
\hline Vital signs assessed by EMT & & \\
\hline Mental status & 419 & 100 \\
\hline RR & 405 & 96.7 \\
\hline Pulse & 401 & 95.7 \\
\hline Oxygen saturation & 297 & 70.9 \\
\hline BP & 283 & 67.5 \\
\hline Blood glucose & 95 & 22.7 \\
\hline Interventions performed by EMT & & \\
\hline Oxygen & 329 & 78.5 \\
\hline Intravenous placed & 194 & 46.3 \\
\hline Intravenous fluids given & 143 & 34.1 \\
\hline Tramadol (pain medication) & 112 & 26.7 \\
\hline Bandages applied & 107 & 25.5 \\
\hline Wound irrigated & 70 & 16.7 \\
\hline Clothing removed & 57 & 13.6 \\
\hline
\end{tabular}

EMT, emergency medical technician.
Table 3 Median TBSA and cumulative 30-day mortality, by intent and by gender

\begin{tabular}{|c|c|c|c|c|c|c|}
\hline \multirow[b]{2}{*}{ Characteristic } & \multicolumn{2}{|c|}{ Survived } & \multicolumn{2}{|c|}{ Died } & \multicolumn{2}{|l|}{ TBSA } \\
\hline & $\mathrm{n}$ & $\%$ & $\mathrm{n}$ & $\%$ & Median & IQR \\
\hline All* & 134 & 35.5 & 243 & 64.5 & 60 & $(30-90)$ \\
\hline Female & 68 & 27.2 & 182 & 72.8 & 70 & $(36-91)$ \\
\hline Male & 66 & 52.0 & 61 & 48.0 & 36 & $(20-70)$ \\
\hline Non-accidental & 25 & 14.3 & 150 & 85.7 & 80 & $(50-100)$ \\
\hline Female & 12 & 9.8 & 111 & 90.2 & 81 & $(60-100)$ \\
\hline Male & 13 & 25.0 & 39 & 75.0 & 70 & $(36-90)$ \\
\hline Accidental & 109 & 54.5 & 91 & 45.5 & 35 & $(17-68)$ \\
\hline Female & 56 & 44.8 & 69 & 55.2 & 40 & $(20-80)$ \\
\hline Male & 53 & 70.7 & 22 & 29.3 & 25 & $(13-45)$ \\
\hline
\end{tabular}

*377 patients were followed up at 30 days; 62 were lost to follow-up. TBSA, total body surface area

Cumulative mortality at 30 days, for all enrolled patients, was $64.5 \%$. The largest proportion of deaths $(34.7 \%, n=131)$ occurred within 24 hours of transport. The revised Baux score performed well (area under the curve 0.92) as a predictor of mortality and the $\mathrm{LD}_{50}$ in this population was a score of 84 (78-89), similar to a score of 80 seen in a previous study in an LMIC. ${ }^{11}$ However, the median score varied by gender and intentionality. The median revised Baux score for men with accidental burns was 65 (40-92) compared with the median of those men with non-accidental burns, 112 (78-144). The median revised Baux score for women with accidental burns was 89 (60-115) compared with the median revised Baux score of those with non-accidental burns, 132 (103-142) Figure 2. (figure 1

TBSA was the strongest predictor of mortality. The unadjusted OR for mortality for burns involving greater than 10\% TBSA was 25.1 (95\% CI 7.5 to 83.8). The $\mathrm{LD}_{50}$ for TBSA burned was $42 \%$. The number of cases enrolled from each state varied widely, and while included in our multivariate regression, state was not a statistically significant predictor. The strongest predictors of mortality were TBSA greater than 10\% (OR 7.9; 95\% CI 2.2 to 28.7 ), inhalation injury (OR $5.5 ; 95 \%$ CI 2.5 to 12.2 ) and intentionality (OR 4.7; 95\% CI 2.5 to 8.8) (table 4). Economic status (OR 2.3; 95\% CI 1.0 to 5.1) and female gender (OR 2.2; $95 \%$ CI 1.2 to 3.9 ) were also predictive of mortality.

\section{DISCUSSION}

In our study, female gender and non-accidental burns were key drivers of mortality. These factors, in addition to time to care, presence of inhalation injury and TBSA, must be considered when addressing the epidemic of burns in India. Additionally, this study demonstrates that EMS can help by providing critical interventions and rapidly transporting patients to care.

Previous studies noted that delays in reaching care were key contributors to poor outcomes. ${ }^{6}{ }^{13-16}$ In this study, patients and families sought medical care emergently. Even when using the most conservative estimates for time from injury to EMS activation and time from that call to hospital arrival, we found that $88 \%$ of patients reached hospital care within 3 hours of injury. This is a significant improvement from the mean of 4 hours from injury to hospital care previously reported. ${ }^{6}$ Patients transported by EMS often received stabilisation care prior to hospital arrival: EMTs provided oxygen, fluids, wound care and pain control in the field. Future goals for this EMS organisation include further increasing the delivery rates of pain medication, oxygen and intravenous fluids, which may require additional specialised 


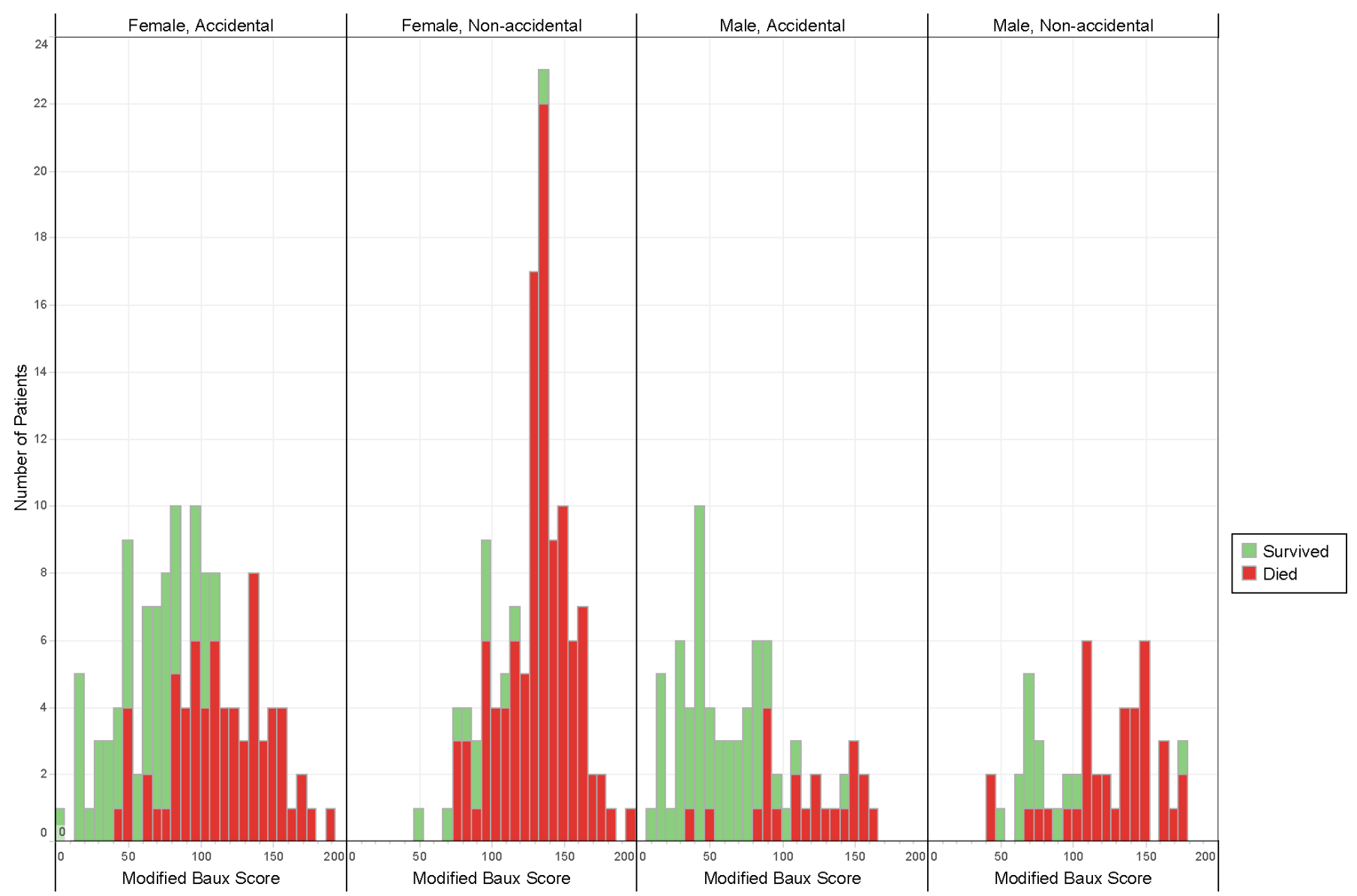

Figure 2 Revised Baux scores, by gender and intentionality, of patients using emergency medical services (EMS) across five states in India.

training in difficult intravenous placement and intraosseous access.

The severity and associated mortality of burns seen in this study contrast sharply with that seen in other countries. A systematic review of severe burn injuries in Europe, defined as those requiring hospital admission, found the average TBSA burned ranged from $11 \%$ to $24 \%$ with a maximum reported mortality of $34 \% .{ }^{17}$ Similarly, in the USA, three-quarters of burns involve less than 10\% TBSA, and the average mortality is $5.7 \% .^{18}$ Thus, Indian EMTs are encountering sicker patients with a much higher burn severity-60\% median TBSA burned and $64.5 \%$

Table 4 Multivariate regression of mortality for patients using EMS for burns across five states in India

\begin{tabular}{lcl}
\hline Variable & Unadjusted OR $(95 \% \mathrm{CI})$ & Adjusted OR $(95 \% \mathrm{Cl})$ \\
\hline Time to hospital & $1.0(0.99$ to 1.0$)$ & $1.0(1.0$ to 1.0$)$ \\
\hline Age & $1.0(1.0$ to 1.0$)$ & $1.0(1.0$ to 1.0$)$ \\
\hline Tertiary hospital care & $1.6(1.0$ to 2.7$)$ & $1.0(0.5$ to 2.1$)$ \\
\hline Rural & $1.0(0.6$ to 1.6$)$ & $1.1(0.5$ to 2.2$)$ \\
Social status & $1.9(1.2$ to 2.9$)$ & $1.7(0.9$ to 3.4$)$ \\
Female gender* & $2.9(1.9$ to 4.5$)$ & $2.2(1.2$ to 3.9$)$ \\
Economic status-below & $1.1(0.7$ to 1.7$)$ & $2.3(1.0$ to 5.1$)$ \\
poverty level* & $7.2(4.3$ to 11.9$)$ & $4.7(2.5$ to 8.8$)$ \\
Non-accidental burn* & $6.0(3.7$ to 9.9$)$ & $5.5(2.5$ to 12.2$)$ \\
Inhalation injury present* & $25.1(7.5$ to 83.8$)$ & $7.9(2.2$ to 28.7$)$ \\
\hline TBSA burned $>10 \%{ }^{*}$ &
\end{tabular}

*Statistically significant predictors in multivariate analysis $(p<0.05)$.

EMS, emergency medical services. mortality. Additionally, the $\mathrm{LD}_{50}$ in the USA is $65 \%-70 \%$ TBSA, substantially higher than that seen in our study $(42 \%) .{ }^{18}$ One explanation may be the higher prevalence of inhalation injury, which is not accounted for when looking at TBSA alone. When comparing median revised Baux scores, which includes inhalation injury, 68\% had Baux scores greater than 80 (the previously established $\mathrm{LD}_{50}$ ). In fact, $75 \%$ of men with non-accidental burns and $93 \%$ of women with non-accidental burns had revised Baux scores greater than 80 . Other reasons for this high mortality in India may be health system factors (eg, density of critical care), social determinants of health (eg, family support and integration, socioeconomic status) and patient factors (eg, coping and resiliency in patients with non-accidental burns).

This study highlights the role of social determinants of health related to burn outcomes through the associations identified between gender, TBSA burned, mortality and intentionality. The high overall mortality from non-accidental burns is similar to that seen in other studies in India. ${ }^{6} 131920$ Outside of India, men are more likely to self-immolate ${ }^{38}$; in India, this action is primarily undertaken by women and is associated with a history of domestic violence, harassment by in-laws and/or depression or other mental health issues. ${ }^{1421}$ Consequently, even those who initially survive their burns may be isolated from their families and struggling with even deeper depression.

When comparing accidental with non-accidental burns across genders, the TBSA burned, revised Baux scores and observed mortality significantly differed. In accidental burns, more women suffered high TBSA burns, which may be related to the sari, a traditional women's dress in India, which is loose fitting and long. In non-accidental burns, there is an exponential growth 
Box 1 American Burn Association's referral criteria

The following burn injuries should be referred to a burn centre:

- Partial-thickness burns of greater than $10 \%$ of the total body surface area.

- Burns that involve the face, hands, feet, genitalia, perineum or major joints.

- Third-degree burns in any age group.

- Electrical burns, including lightning injury.

- Chemical burns.

- Inhalation injury.

- Burn injury in patients with pre-existing medical disorders that could complicate management, prolong recovery or affect mortality.

- Any patients with burns and concomitant trauma (such as fractures) in which the burn injury poses the greatest risk of morbidity or mortality. In such cases, if the trauma poses the greater immediate risk, the patient's condition may be stabilised initially in a trauma centre before transfer to a burn centre. Physician judgement will be necessary in such situations and should be in concert with the regional medical control plan and triage protocols.

- Burned children in hospitals without qualified personnel or equipment for the care of children.

- Burn injury in patients who will require special social, emotional or rehabilitative intervention.

in the TBSA curve above $30 \%$ for women, whereas with men, the overall slope is relatively flat. Considering all burns, women are twice as likely to die from their burns, even when adjusting for TBSA involved and intentionality. In addition, severe self-inflicted burns were equally present above and below the poverty level in this study; yet, poorer patients were over twice as likely to die.

EMS connects patients to advanced medical care that is essential for the severely burned population. In the USA, transfer of patients to burn centres is guided by American Burn Association's referral criteria ${ }^{22}$ (box 1). Most patients in this study would require transfer to burn centres based on these criteria, including the need for 'special social, emotional, or rehabilitative intervention' given the high prevalence of non-accidental burns. It is likely that EMTs are already bypassing closer hospitals to transport patients to higher levels of care. Only 3.6\% of ambulances transported patients to primary care centres, travelling a median distance of $8.5 \mathrm{~km}$ (IQR: 3-12); whereas $58.4 \%$ were transported to secondary care centres and $27.2 \%$ were transported to tertiary care centres, requiring travel of twice the distance: $17 \mathrm{~km}$ (IQR: 8-27) and $10 \mathrm{~km}$ (IQR: 5-22), respectively. Developing transport protocols based on local capacity and using available information such as TBSA burned, suspected inhalation injury and vital signs could help direct EMTs to the appropriate initial medical facility, which may not necessarily be a burn centre.

\section{Limitations}

First, our conclusions are limited by the number of patients lost to follow-up. Of the 62 patients lost to follow-up, $48.4 \%(n=30)$ suffered non-accidental injuries, with a median TBSA of 50\% (IQR 20-70). We are likely underestimating mortality. Second, we may be limited by our reliance on self or family report regarding the cause of the burn. We are likely underestimating the prevalence of non-accidental burns, whether self-inflicted or due to assault, in this population, as patients or families may be afraid or ashamed to disclose the mechanism. ${ }^{23}$ This study signals the need to develop a systematic approach to investigating the cause of burns at the healthcare system level. The WHO guidelines for confidential enquiries in maternal death reviews may serve as a model to better understand the roots of this devastating public health issue. ${ }^{21}$

Finally, our study design was limited by practical constraints allowing only for a convenience sample from daytime EMS calls. While our sample represents over $10 \%$ of all calls for burns across five states, we were unable to include late evening or overnight calls. As a result, we may be underestimating burns related to gender-based violence because there may be a higher incidence of this violence after men return home.

\section{Policy implications}

There are an estimated 700000 admissions annually for burns in India, but only $800-1000$ beds in specialised burn units in the entire country. ${ }^{24}$ Consequently, this precludes EMS protocols prioritising delivery of patients to centres with specialised burn care capacity. Many patients must travel long distances to reach this specialty care. Therefore, it is equally important that public health investment includes EMS infrastructure that can stabilise patients with critical burns and connect them to definitive care. Swanson and colleagues found that $75 \%$ of burn-related deaths occur within the first 72 hours, predominately due to shock, lung injury and anoxic brain injury. ${ }^{15} 1625$ The prevention and management of each of these conditions can begin in the prehospital setting with airway management, oxygenation, avoidance of hypothermia and early fluid management.

Moreover, with burns as severe as seen in our study, prevention will likely have the greatest impact. The WHO Plan for Burn Prevention and Care, like many other approaches, emphasises prevention in the form of smoke detectors, flame-resistant clothing and changes to cooking practices. ${ }^{26}$ Unfortunately, the Plan does not mention mental health and gender-based violence support services. In India, the lifetime prevalence of genderbased violence is $37.2 \%$ and less than $1 \%$ of women will ever reach out for help beyond their family and friends. ${ }^{27}$ We demonstrate that non-accidental burns carried the highest severity and mortality, and this cohort was primarily young, married women. Consequently, our study emphasises the need to consider the role of mental health and gender-based violence services as integral to the prevention of burns.

Patients and families use EMS for patients with severe burns in India. EMS can initiate treatment and rapidly connect patients with critical illness to higher level care. Despite this, mortality remains high, with women disproportionality suffering non-accidental burns and higher mortality. Future efforts must focus on rapid prehospital medical care and critical care development, as well as on a burn prevention strategy that includes mental health and gender-based violence supportive services.

Acknowledgements Special thanks to Loretta Matheson for her help with statistical review; and to Aruna Gimkala, Royal Uddin Ahmed, Steffy Christian, Roshidul Islam, Chetana Jani, Rupjoy Maibangsa, Hanamesh Nagappa, Divya Patel, Niyati Patel, Om Raj, Srinivas Rao, Marada Lakshmana Rao, Geetanjali Thakur, Hem Prakash Thakur, Isberth Tham and Munirathnamama Venkateshappa for their help with data collection and data quality monitoring

Contributors JAN and MCS wrote the first draft of the article. JAN and EAP conducted the statistical analysis. JAN, CBB, EAP, GVRR, SVM and MCS contributed to study design. All authors assisted in interpretation of findings and revision of the manuscript. All authors read and approved the final manuscript. 
Funding The work was funded jointly by Stanford University and GVK EMRI as part of collaborative agreement. No external funding supported this project.

Competing interests GVRR is the Director of Research and the Emergency Management Learning Centre at GVK EMRI.

Patient consent Not required.

Ethics approval The Institutional Review Board at Stanford deemed the study exempt because data were collected as part of routine quality improvement processes. The Ethics and Research Committee at GVK EMRI approved the publication of these findings.

Provenance and peer review Not commissioned; externally peer reviewed.

Open access This is an open access article distributed in accordance with the Creative Commons Attribution Non Commercial (CC BY-NC 4.0) license, which permits others to distribute, remix, adapt, build upon this work non-commercially, and license their derivative works on different terms, provided the original work is properly cited, appropriate credit is given, any changes made indicated, and the use is non-commercial. See: http://creativecommons.org/licenses/by-nc/4.0/.

\section{REFERENCES}

1 Global Burden of Disease Study 2013 Collaborators. Global, regional, and national incidence, prevalence, and years lived with disability for 301 acute and chronic diseases and injuries in 188 countries, 1990-2013: a systematic analysis for the Global Burden of Disease Study 2013. Lancet 2015;386:743-800.

2 GBD 2013 Mortality and Causes of Death Collaborators. Global, regional, and national age-sex specific all-cause and cause-specific mortality for 240 causes of death, 1990-2013: a systematic analysis for the Global Burden of Disease Study 2013. Lancet 2015;385:117-71.

3 Peck MD. Epidemiology of burns throughout the world. Part I: Distribution and risk factors. Burns 2011;37:1087-100

4 Forjuoh SN. Burns in low- and middle-income countries: a review of available literature on descriptive epidemiology, risk factors, treatment, and prevention. Burns 2006;32:529-37.

5 Sanghavi P, Bhalla K, Das V. Fire-related deaths in India in 2001: a retrospective analysis of data. Lancet 2009;373:1282-8.

6 Ganesamoni S, Kate V, Sadasivan J. Epidemiology of hospitalized burn patients in a tertiary care hospital in South India. Burns 2010;36:422-9.

7 Kumar S, Ali W, Verma AK, et al. Epidemiology and mortality of burns in the Lucknow Region, India-a 5 year study. Burns 2013;39:1599-605.

8 Laloë V, Beer-sheva I, Farah J, Amman J, Asia FE. Patterns of deliberate self-burning in various parts of the world. A review. Burns 2004;30:207-15.
9 Allgaier RL, Laflamme L, Wallis LA. Operational demands on pre-hospital emergency care for burn injuries in a middle-income setting: a study in the Western Cape, South Africa. Int J Emerg Med 2017;10:2.

10 Harris PA, Taylor R, Thielke R, et al. Research electronic data capture (REDCap)-a metadata-driven methodology and workflow process for providing translational research informatics support. J Biomed Inform 2009;42:377-81.

11 Osler T, Glance LG, Hosmer DW. Simplified estimates of the probability of death after burn injuries: extending and updating the baux score. J Trauma 2010;68:690-7.

12 Smith MT, Allorto NL, Clarke DL. Modified first world mortality scores can be used in a regional South African burn service with resource limitations. Burns 2016;42:1340-4.

13 Ahuja RB, Bhattacharya S. An analysis of 11,196 burn admissions and evaluation of conservative management techniques. Burns 2002;28:555-61.

14 Natarajan M. Differences between intentional and non-intentional burns in India: implications for prevention. Burns 2014;40:1033-9.

15 Latenser BA. Critical care of the burn patient: the first 48 hours. Crit Care Med 2009;37:2819-26.

16 Pham TN, Gibran NS. Thermal and electrical injuries. Surg Clin North Am 2007;87:185-206.

17 Brusselaers N, Monstrey S, Vogelaers D, et al. Severe burn injury in Europe: a systematic review of the incidence, etiology, morbidity, and mortality. Crit Care 2010;14:R188.

18 American Burn Association. National Burn Repository Report of Data from 20052015, 2015. www.ameriburn.org/2015NBRAnnualReport.pdf. (Accessed 10 Nov 2017).

19 Jayaraman V, Ramakrishnan KM, Davies MR, et al. India: an analysis of 1368 patients in 1 year. Burns 1993;19:339-44.

20 Subrahmanyam M. Epidemiology of burns in a district hospital in western India. Burns 1996;22:439-42.

21 Batra AK. Burn mortality: recent trends and sociocultural determinants in rural India. Burns 2003;29:270-5.

22 American Burn Association/American College of Surgeons. Guidelines for the operation of burn centers. J Burn Care Res 2007;28:134-41.

23 World Health Organization. Beyond the numbers: reviewing maternal deaths and complications to make pregnancy safer. Br Med Bull 2004;67:27-37.

24 Sachan D. India launches major programme to treat burn injuries. BMJ 2014;348:g1658.

25 Swanson JW, Otto AM, Gibran NS, et al. Trajectories to death in patients with burn injury. J Trauma Acute Care Surg 2013;74:282-8.

26 Mock C, Peck M, Krug E, et al. Confronting the global burden of burns: A WHO plan and a challenge, 2009.

27 Palermo T, Bleck J, Peterman A. Tip of the iceberg: reporting and gender-based violence in developing countries. Am J Epidemiol 2014;179:602-12. 